\title{
Potential drawbacks of frequent asymptomatic coronavirus disease 2019 (COVID-19) testing
}

\author{
Giuseppe Lippi MD ${ }^{1}$ (1), Brandon M. Henry MD²,a and Fabian Sanchis-Gomar MD 3,a \\ ${ }^{1}$ Section of Clinical Biochemistry, University of Verona, Verona, Italy, ${ }^{2}$ Cardiac Intensive Care Unit, The Heart Institute, Cincinnati Children's Hospital Medical \\ Center, Ohio, United States and ${ }^{3}$ Department of Physiology, Faculty of Medicine, University of Valencia and INCLIVA Biomedical Research Institute, Valencia, \\ Spain
}

To the Editor-Although significant emphasis has been given to widespread community screening for identifying severe acute respiratory syndrome coronavirus 2 (SARS-CoV-2) infection, also in asymptomatic people, Bai et $\mathrm{al}^{1}$ recently concluded that this strategy would not add clinically useful information, nor would have significant impact on current infection control management. The theoretical benefits of population coronavirus disease 2019 (COVID-19) screening include achieving more information for forecasting pandemic evolution, optimizing and quickening the establishment of preventive and containment strategies, and accurately and rapidly assessing the efficiency of implemented measures. ${ }^{2}$ On the other hand, there are also some potential drawbacks that may emerge from mass testing of asymptomatic patients.

Currently, reagent availability is the most limiting aspect for implementation of large-scale testing. A recent survey of the American Association of Clinical Chemistry (AACC) has revealed that $>50 \%$ of worldwide clinical laboratories were still facing dramatic shortages of test kits and reagents at the end of September 2020 , with $>70 \%$ of respondents emphasizing substantial challenges to increase their testing capacity. ${ }^{3}$ The gold standard for diagnosing COVID-19 is identification of viral RNA in upper respiratory tract samples, collected and tested by skilled and trained healthcare personnel. Thus, staff shortages will further complicate the possibility of amplifying the actual testing volume, which remains now insufficient for even testing all suspect and symptomatic subjects in many worldwide regions. Therefore, specimen collection and reagent shortages have inhibited rapid increases in testing capacity, representing a bottleneck and critical limitation in intensifying SARS-CoV-2 diagnostic testing.

The widespread identification of several hundred thousand, or even millions, of asymptomatic people, representing now the vast majority of SARS-CoV-2-positive cases in certain regions, is a second important aspect. It is now undeniable that the infectivity of asymptomatic COVID-19 patients is weaker and progressively declines over time. ${ }^{4}$ Therefore, mandatory isolation of a massive number of people, who are less likely to substantially contribute to transmitting the virus even when positive (the secondary attack rate of asymptomatic people has been reported at around 3\%), ${ }^{5}$ especially when all the recommended preventive measure are adopted (ie, social distancing, hand hygiene, use of face masks, avoid singing or shouting), will likely bring forth further negative

\footnotetext{
Author for correspondence: Prof. Giuseppe Lippi, Email: giuseppe.lippi@univr.it

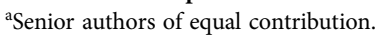

Cite this article: Lippi G, Henry BM, and Sanchis-Gomar F. (2021). Potential drawbacks of frequent asymptomatic coronavirus disease 2019 (COVID-19) testing. Infection Control \& Hospital Epidemiology, 42: 1405-1406, https://doi.org/10.1017/ ice. 2020.1305
}

Table 1. Potential Drawbacks of Widespread Asymptomatic COVID-19 Testing

- Shortage of supplies and human resources for testing symptomatic patients and for contact tracing

- Unnecessary isolation of subjects with non-progressive disease and lower infectivity

- Adverse economic, societal and healthcare consequences - Physiological distress

- Risk of overdiagnosis and overtreatment

impacts on society, economy, and even healthcare, whereby isolation of many asymptomatic physicians and other healthcare providers would impair the possibility to deliver standard care. ${ }^{6}$ There are also important psychological consequences from the quarantining of asymptomatic COVID-19 subjects, who may develop a wide array of disturbances such as psychological distress and declining daily functioning. ${ }^{7}$ Moreover, questions arise as to whether "high-risk" contacts of asymptomatic individuals (ie, household family members, etc), would also require quarantine, further complicating a mass testing strategy.

Finally, diagnosing asymptomatic COVID-19 people, who are unlikely to develop medium- or long-term consequences from this infection, could expose these people to further invasive and potentially harmful testing (eg, radiation) and even unnecessary treatments, which may be associated with undesirable side effects that could be worse than the disease itself. ${ }^{8,9}$ Rates of false-negative swab tests between $2 \%$ and $33 \%$ in repeat sample testing have been reported, while rates of false positives have been estimated between $0.8 \%$ and $4.0 \%$, probably due to technical problems such as contamination during sampling, contamination of amplicons or reagents, and cross reactions with other viruses or genetic material. ${ }^{10}$ Assuming a false-positive rate of only $1 \%$, for every 1 million tests run per day, 10,000 false-positive results would occur. This, combined with the detection of tens of thousands of asymptomatic individuals daily at lower risk of transmitting the virus in the presence of appropriate precautions, would likely overwhelm our ability to effectively contact trace and rapidly contain the highest-risk clusters.

No evidence-based data for universal screening of asymptomatic COVID-19 patients has been reported so far. Although we agree that strengthened molecular and/or antigenic COVID19 testing of symptomatic subjects and their contacts represents a mainstay for pandemics containment, additional evidence and development of new public health strategies to handle the results of such testing would be needed before massive testing of

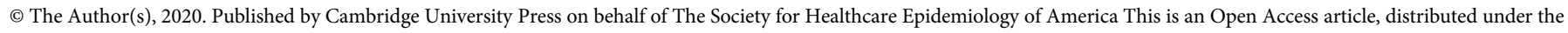

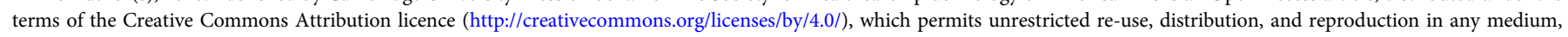
provided the original work is properly cited. 
asymptomatic COVID-19 individuals could be recommended (Table 1). Importantly, testing nonsymptomatic individuals may also cause false sense of security. To date, universal precautions such as hand and respiratory hygiene, self-quarantine when symptomatic or possible contact, social distancing, and use of masks are the best methods to mitigate COVID-19.

\section{Acknowledgments.}

Financial support. No financial support was provided relevant to this article.

Conflicts of interest. All authors report no conflicts of interest relevant to this article.

\section{References}

1. Bai AD, Li XX, Alsalem M, et al. Utility of asymptomatic inpatient testing for COVID-19 in a low-prevalence setting: a multicenter point-prevalence study. Infect Control Hosp Epidemiol 2020;41:1233-1235.

2. Müller M, Derlet PM, Mudry C, Aeppli G. Testing of asymptomatic individuals for fast feedback-control of COVID-19 pandemics. Phys Biol 2020. doi: 10.1088/478-3975/aba6d0.
3. Coronavirus testing survey. American Association of Clinical Chemistry website. https://www.aacc.org/science-and-research/covid-19-resources/ aacc-covid-19-testing-survey. Accessed October 14, 2020.

4. Walsh KA, Spillane S, Comber L, et al. The duration of infectiousness of individuals infected with SARS-CoV-2. J Infect 2020. doi: 10.1016/j.jinf. 2020.10.009.

5. Zhang W, Cheng W, Luo L, et al. Secondary transmission of coronavirus disease from presymptomatic persons, China. Emerg Infect Dis 2020;26:1924-1926.

6. Thornton J. COVID-19: delays in getting tests are keeping doctors from work, health leaders warn. BMJ 2020;370:m3755.

7. Upadhyay R, Sweta, Singh B, Singh U. Psychological impact of quarantine period on asymptomatic individuals with COVID-19. Soc Sci Human Open 2020;2:100061.

8. Cella L, Gagliardi G, Hedman M, Palma G. Injuries from asymptomatic COVID-19 disease: new hidden toxicity risk factors in thoracic radiation therapy. Int J Radiat Oncol Biol Phys 2020;108:394-396.

9. Gao Z, Xu Y, Sun C, et al. A systematic review of asymptomatic infections with COVID-19. J Microbiol Immunol Infect 2020. doi: 10.1016/j.jmii.2020. 05.001 .

10. Surkova E, Nikolayevskyy V, Drobniewski F. False-positive COVID-19 results: hidden problems and costs. Lancet Respir Med 2020. doi: 10. 1016/S2213-2600(20)30453-7.

\title{
Clinical characteristics and persistence of severe acute respiratory coronavirus virus 2 (SARS-CoV-2) IgG antibodies in 4,607 French healthcare workers: Comparison with European countries
}

\author{
Chantal Delmas MD¹, Genevieve Plu-Bureau MD, PhD², Etienne Canouï MD³ , Luc Mouthon MD, $\mathrm{PhD}^{4}$ and \\ Jean-Francois Meritet $M D^{5}$ \\ ${ }^{1}$ Occupational Health Department, GH Paris Centre - Cochin, APHP, France, ${ }^{2}$ Epopee Team Inserm U1153 and Medical Gynecology Unit, GH Paris Centre - Cochin \\ APHP, University of Paris, Paris, France, ${ }^{3}$ Antimicrobial Stewardship Team, GH Paris Centre - Cochin, APHP, Paris, France, ${ }^{4}$ Internal Medicine Department, GH \\ Paris Centre - Cochin, APHP, University of Paris, Paris, France and ${ }^{5}$ Virology Department, GH Paris Centre - Cochin APHP, Paris France
}

To the Editor-The safety of healthcare workers (HCWs) is a major challenge for healthcare systems. In the course of a severe acute respiratory coronavirus virus 2 (SARS-CoV-2) infection, immunoglobulin G (IgG) antibodies may be detected after a median of 14-24 days (interquartile range [IQR], 10-18) after onset of symptoms. ${ }^{1}$

In France, the coronavirus disease 2019 (COVID-19) pandemic reached a peak on April 7, 2020. HCWs had mobility and flexibility inside the Paris Center university hospital, where there was a cluster in the pandemic. We investigated the prevalence of IgG antibodies against SARS-CoV-2 among all HCWs in this hospital. We also sought to determine the correlation between RT-PCR test and serology and to compare our seroprevalence with that of other European countries.

From May 14, 2020, to June 17, 2020, all HCWs were asked by the occupational health department to participate in serologic screening.

\footnotetext{
Author for correspondence: Chantal Delmas, E-mail address: chantal.delmas@aphp.fr Cite this article: Delmas C, et al. (2021). Clinical characteristics and persistence of severe acute respiratory coronavirus virus 2 (SARS-CoV-2) IgG antibodies in 4,607 French healthcare workers: Comparison with European countries. Infection Control \& Hospital Epidemiology, 42: 1406-1408, https://doi.org/10.1017/ice.2020.1309
}

The Abbott-Architect test (Abbott Laboratories, Abbott Park, IL) was used to detect IgG anti-SARS-CoV-2. During blood sampling, clinical information was recorded using a standardized self-questionnaire on presented symptoms, comorbidities, and the reversetranscriptase polymerase chain reaction (RT-PCR) test if one had been previously performed. Blood samples were collected $>28$ days after the first symptoms from those who were symptomatic.

The seroprevalence and $95 \%$ confidence interval were estimated using the Fisher exact method. The $t$ test and the $\chi^{2}$ test were performed to compare quantitative and qualitative variables, respectively. Simple and multivariate logistic regressions were performed to assess risk and symptoms associated with seroprevalence respectively. Statistical analyses were performed using SAS software (SAS Institute, Cary, NC). The local institutional review board approved this study. All subjects participated voluntarily under pseudonyms.

Of 5,021 workers present during the study period, 4,607 $(91.8 \%)$ were included in the study. The mean age was 41.8 years (SD, 12.6), and $75 \%$ were female. Furthermore, $45 \%$ were paramedical staff members, $36 \%$ were physicians (including medical students), and 19\% were in administrative and other professions. 